\title{
ARE THERE ANY RISK FACTORS FOR DEVELOPING COMPLICATIONS WITH THE USE OF RETRIEVABLE VENA CAVA FILTERS IN ORTHOPAEDIC SURGERY?
}

\author{
¿EXISTEN FACTORES DE RIESGO PARA DESARROLLAR COMPLICACIONES CON EL USO DE \\ FILTROS DE VENA CAVA REMOVIBLES EN CIRUGÍAS ORTOPÉDICAS?
}

\author{
Pablo A. I. Slullitel ${ }^{1,4}$, Fernando Díaz Dilernia ${ }^{1}$, Joaquín Stagnaro ${ }^{1}$, Mariano Revah $^{1}$, Liliana P. Rojas ${ }^{2}$, \\ María L. Posadas-Martínez², Martín A. Buttaro ${ }^{1}$, Gastón A. Slullitel',3.
}

\begin{abstract}
:
Background: Thromboprophylaxis may be insufficient to prevent thromboembolic events in high risk patients after orthopaedic procedures. In this scenario, a retrievable vena cava filter (VCF) could be considered as an alternative, although it's use remains controversial. Aim: To estimate mortality, mechanical and hematological complications associated with the use of retrievable VCFs in orthopaedic surgery Methods: Retrospective cohort of patients with history of thromboembolic (TED) disease who underwent orthopaedic surgery and a retrievable VCF was placed, between 2006-2014 at Hospital Italiano de Buenos Aires. Permanent filters were excluded. The main outcomes were filter's mechanical complications, hematologic complications (TED recurrence, post-thrombotic syndrome and major bleeding) and death. To estimate association with risk factors, we subclassified surgeries into 5 groups: 1, arthroplasty/non-arthroplasty; 2, primary/revision; 3, elective/urgent; 4, oncologic/non-oncologic; 5, preoperative/postoperative filter. Results: Sixty eight patients were included, of those 31 presented a complication. Mechanical complications were $16 \%$ and required a filter revision. Sixty-four percent of the revised VCFs developed a mechanical failure and could not be retrieved. Overall prevalence of TED recurrence, post-thrombotic syndrome and hemorrhage was 33\%, 15\% and $4.5 \%$, respectively. Spinal surgeries were a risk factor for developing TED recurrences. The mortality rate was $28 \%$ and death related with TED recurrence $4 \%$. Conclusions: Orthopaedic procedures had a high risk of mechanical and hematologic complications after using a retrievable VCF. However, mortality was low due to these complications.
\end{abstract}

Keywords: retrievable vena cava filter; thromboembolic disease; orthopedic surgery; deep vein thrombosis; pulmonary embolism

\section{Resumen:}

Introducción: En pacientes sometidos a cirugía ortopédica y con antecedente de Enfermedad tromboembolica, la profilaxis común suele ser insuficiente para prevenir eventos tromboembólicos. Los filtros de vena cava (FVC) removibles pueden considerarse una alternativa. Objetivos: Estimar la tasa de complicaciones hematológicas, mecánicas y muertes asociadas al uso de FVC removibles en cirugía ortopédica. Métodos: Se diseñó una cohorte retrospectiva de pacientes con historia previa de Enfermedad tromboembolica (ETE) sometidos a procedimientos ortopédicos que requirieron FVC removible, entre el 2006-2014 en el servicio de ortopedia del Hospital Italiano de Buenos Aires. Se definió complicación asociada al FVC a las complicaciones mecánicas, hematológicas (recurrencia de ETE, síndrome postrombotico y sangrado mayor) y muerte. Para estimar la asociación con factores de riesgo, subclasificamos a las cirugías en 5 grupos: 1, artroplastia/no artroplastia; 2, primaria/revisión; 3 , electiva/urgente; 4, oncológica/no oncológica; 5, filtro pre/postoperatorio. Resultados: Se incluyeron 68 pacientes, de los cuales 31 presentaron algún tipo de complicación. Las complicaciones mecánicas ostentaron un $16 \%$, precisando de una revisión del filtro. $64 \%$ de los filtros revisados fallaron mecánicamente y no pudieron ser extraídos. Las tasas de recurrencia de ETE, síndrome postrombotico y sangrado mayor fueron del $33 \%, 15 \%$ y $4.5 \%$, respectivamente. Las cirugías espinales presentaron un mayor riesgo de recurrencia de ETE. La mortalidad global fue del $28 \%$ y $4 \%$ asociada a recurrencia de ETE. Conclusiones: Las cirugías ortopédicas exhibieron un riesgo elevado de complicaciones mecánicas y hematológicas luego de usar un FVC removible. Empero, la mortalidad debido a dichas complicaciones fue baja.

Palabras Clave: filtro de vena cava removible; enfermedad tromboembólica; cirugía ortopédica; trombosis venosa profunda; embolia pulmonar.

\footnotetext{
1 "Carlos E. Ottolenghi" Institute of Orthopedics, Italian Hospital of Buenos Aires, Buenos Aires, Argentina.

2 Internal Medicine Research Unit, Italian Hospital of Buenos Aires, Buenos Aires, Argentina.

3 Institute of Orthopaedics "Dr. Jaime Slullitel," Rosario, Santa Fe, Argentina.

4 Email de contacto: pablo.slullitel@gmail.com
} 


\section{Introduction}

Thromboembolic disease (TED) is a frequent complication following orthopaedic procedures with a prevalence of almost $50 \%$ in the absence of any means of antithrombotic prophylaxis, ${ }^{1}$ although modern rates are thought to be much lower. ${ }^{2}$ In high-risk patients, such as those with previous or acute TED, coagulopathies, oncologic, high-grade congestive heart failure, atrial fibrillation or polytraumatized; common prophylaxes may be insufficient or contraindicated to prevent new thrombotic events. ${ }^{3}$

In this scenario, interfering with the venous flow with a vena cava filter (VCF) may be a reliable alternative. VCFs, introduced percutaneously, are metal alloy devices that mechanically trap fragmented thromboemboli from the deep leg veins in route to the pulmonary circulation. Given that most of the complication-related literature is composed of case reports, their exact complication rate is probably under-reported. ${ }^{4}$ Nevertheless, it is widely believed that most filter's complications can be avoided by prompt removal: as time from implantation elapses, chances for a TED recurrence persist or may be even aggravated ${ }^{5}$.

Consequently, retrievable filters are preferred over permanent ones nowadays, although there is a marked paucity of evidence describing their association with mechanical and hematologic complications in orthopaedic surgery ${ }^{6-7}$. The AAOS clinical practice guideline for preventing thromboembolic disease in patients undergoing elective hip and knee arthroplasty was unable to recommend for or against the use of filters because of inconclusive evidence ${ }^{8}$. This issue relies probably on the absence of neither formal clinical guidelines nor high quality evidence to support their indication. Seemingly, current reports of VCF use in orthopaedics are not updated since they mainly derive from an analysis of permanent filters ${ }^{9-8}$.

To our knowledge, it remains controversial whether retrievable VCFs are actually beneficial for orthopaedic patients in whom pharmacological prophylaxis is contraindicated or deficient. Still, orthopedic procedures vary in complexity and certain surgeries are more likely to be associated with thrombogenesis, like arthroplasties; ${ }^{10-11}$ we had hypothesized that patients undergoing arthroplasties, especially those performed urgently secondary to fractures, would have more overall complications related to the VCF than other kind of orthopaedic procedures.

Our aim was to estimate mortality and complications (mechanical and haematologic) of retrievable VCFs within orthopaedic surgery.

\section{Methods}

Design: Retrospective cohort of all consecutive adult patients with history of thromboembolic disease who underwent ortophedic surgery and received an inferior VCF between 2006 and 2014 at Italian Hospital of Buenos Aires. Patients were excluded if a permanent filter was placed, if they were operated by nonorthopaedic services, or if they were duplicate cases.

Indication for VCF was defined by the hematologist based on clinical criteria. General indications for transient filter use were: high-risk patients with contraindication to antithrombotic chemoprophylaxis and patients with suspicion of insufficient prophylaxis once already indicated ${ }^{12}$. In all cases a retrievable Günther Tulip ${ }^{\circledR}$ (Cook Medical, Bloomington, IN, USA) VCF was positioned at the infra-renal vena cava via the femoral vein. A trained angiographist was in charge of the procedure by using a standardized protocol. After accessing the femoral vein using real-time ultrasound, a 5-French pigtail catheter (Angiodynamics, Queensbury, New York) was advanced over a standard guide wire under fluoroscopic control. Once at the proper location, the filter was advanced through a 12-French introducer sheath and then gently deployed. After placement, a coadjuvant therapy with subcutaneous enoxaparin at a prophylactic dose was indicated if patients did not have a contraindication. After filter retrieval and without a consistent protocol, patients continued to receive chemoprophylaxis with either enoxaparin or switched to oral anticoagulation for undefined time.

This study was observational, and all diagnostic and therapeutic medical decisions reflected current medical practice. This study was approved by the ethics committee of the Italian Hospital of Buenos Aires. 


\section{Variables and definitions:}

Main outcomes:

- Hematologic complications: TED recurrence, post-thrombotic syndrome and major bleeding. We defined TED recurrence as a new diagnosis of deep vein thrombosis, through Doppler ultrasound, at any anatomic level of the circulatory venous system or any pulmonary embolus detected by angiotomography after the filter was initially positioned. We registered the date of the thrombotic and/or embolic event. Postthrombotic syndrome was identified whenever signs and symptoms of chronic venous insufficiency secondary to venous hypertension and valvular incompetence were evidenced ${ }^{13}$. Finally, major bleeding was appointed if hemorrhage of the surgical site caused hemodynamic instability or a hemoglobin decline of $2 \mathrm{~g} / \mathrm{dL}$ or more ${ }^{14}$.

- Mechanical complications: Related to the placement or extraction of the filter. Filter malposition was defined when the tip of the filter was not at or near the level of the renal vein inflow. Defective filter deployment was described as an incomplete expansion into the inferior vena cava. Filter migration was considered when the filter shifted to another part of the inferior vena cava, to the heart or to the pulmonary outflow tract. Filter fracture is named to a so. VCF incorporation into the vessel wall was defined as an embedding into endothelium at any portion of the filter, usually diagnosed during the extraction attempt. Finally, filter tilting was defined when it flexed more than 15 degrees from midline. In case any mechanical complication was detected, the requirement of a filter angiographic revision was computed. In all cases, an extraction attempt of the filter was always indicated, following a standard protocol.

- Mortality: Censored at the end of the study on March 2016.

Follow-up was performed through medical electronic records to assess orthopedic complications, and both through medical electronic records and telephonic interview to assess mortality and hematological complications

Demographic data were obtained from IRVTD registry and variables related to surgery were retrieved from the electronic clinical records (ECR). We described the demographic characteristics of all patients including age, gender and major comorbidities. We classified surgeries into 5 subgroups: 1, arthroplasty or nonarthroplasty; 2, primary or revision; 3 , elective or urgent; 4 , oncologic or non-oncologic; 5 , with pre 0 postoperative filter. We also distributed them by anatomical location. We considered a revision surgery as a reoperation performed to correct undesirable sequelae of a previous surgery, with or without an addition or removal of implant components ${ }^{15}$. We defined VCF as pre or postoperative filter and measured time from VCF placement to surgery.

Statistical methods:

Descriptive analysis for categorical variables are expressed as absolute number and percentage, and continuous variables as mean and standard deviation or median and interquartile range, according to the observed distribution. Comparisons between groups were performed with the chi-square test for categorical variables and the Mann-Whitney $U$ test for continuous variables.

Time to filter retrieval and thromboembolic recurrence (DVT and/or PE) since VCF placement were calculated with the Kaplan-Meier estimator and median survival time was expressed with its $95 \%$ confidence interval.

A two-sided $\mathrm{P}<0.05$ was considered significant for all analysis. Statistical analysis was performed using STATA 13.0 .

\section{Results}

\section{Participants}

We computed 186 eligible subjects that received an inferior VCF between 2006 and 2014. Of them, we excluded 118 for having permanent filters, being operated by non-orthopaedic services and/or duplicate cases (Figure 1). Thus, we finally included 68 patients in whom a retrievable VCF was collocated with a 
minimum follow-up of 90 days. All of them had a history of previous thromboembolic event with a retrievable VCF placed within two weeks (before or after) of an orthopaedic surgical procedure. Fifty-four patients $(80 \%)$ had a history of acute or chronic DVT whereas 26 (39\%) had been diagnosed with PE. Additionally, 6\% $(n=5)$ of patients had a previously diagnosed coagulopathy

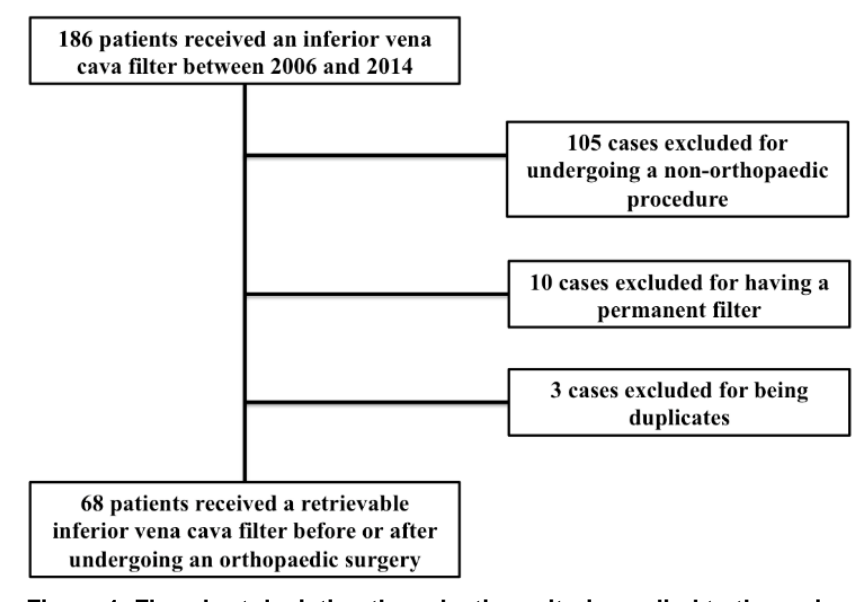

The mean age was 72 (SD 16) years old and the $57 \%$ (39/68) were female. Median time of follow-up was 592 days (IQR: 116-1145).

The prevalence order of surgical procedures was as follows: $57 \%$ hip (39), $17 \%$ knee (12), $13 \%$ midshaft femur (9), 13\% spine (9) and others (16). Sixty one percent (42) of the patients received vena cava filter preoperative and underwent their orthopaedic procedure at an average of 6 days (2-14) after VCF's insertion; those with postoperative filters $(n=26)$ had undergone surgery at an average of 4 days (range, 1-6) before. In $45 \%$ of patients $(n=31)$ non-arthroplasties were performed; the remnant $55 \%(n=37)$ belonged to hip or knee joint replacements. Additionally, $31 \%(n=21)$ of the total surgeries were elective whilst $69 \%(n=47)$ were done urgently. Sixty-five percent of patients $(n=44)$ underwent primary procedures and $35 \%(n=24)$ consisted of revision surgeries. Finally, $30 \%(n=21)$ of cases involved oncologic patients; all of them diagnosed with stage IV disease.

\section{Complications associated with VCF}

Mechanical complications were seen in 11 (16\%) cases: filter malpositioning observed in 9 cases (14.7\%) and tilting in $3(4.4 \%)$. All of them required a filter revision at the angiography operating room. Mean time to filter revision was 96 hours (range; 8-120 hours). Fourteen patients (21\%) developed a mechanical failure, being filter incorporation into the vessel wall the main cause, seen in all but one case. The other filter had a two-fragment fracture during its extraction attempt; as one of the fragments could not be extracted; it was left 'in-situ' requiring implantation of an additional filter proximally. Of the mechanical failures, $64 \%$ of them had a revision previously.

Forty-seven patients had successful filter retrieval (Figure 2). The success rate estimate for filter removal was 69.12\% (CI95\%: 58.03-79.61) and the median time estimate to filter removal was 17.31 days (CI95\%: 12.52-31.34). Patients who died before the first attempt to remove the filter were censored. Figure 2. Graph estimating time to filter retrieval, depicting a median time of 17 days (IC95\%: 13-31). 


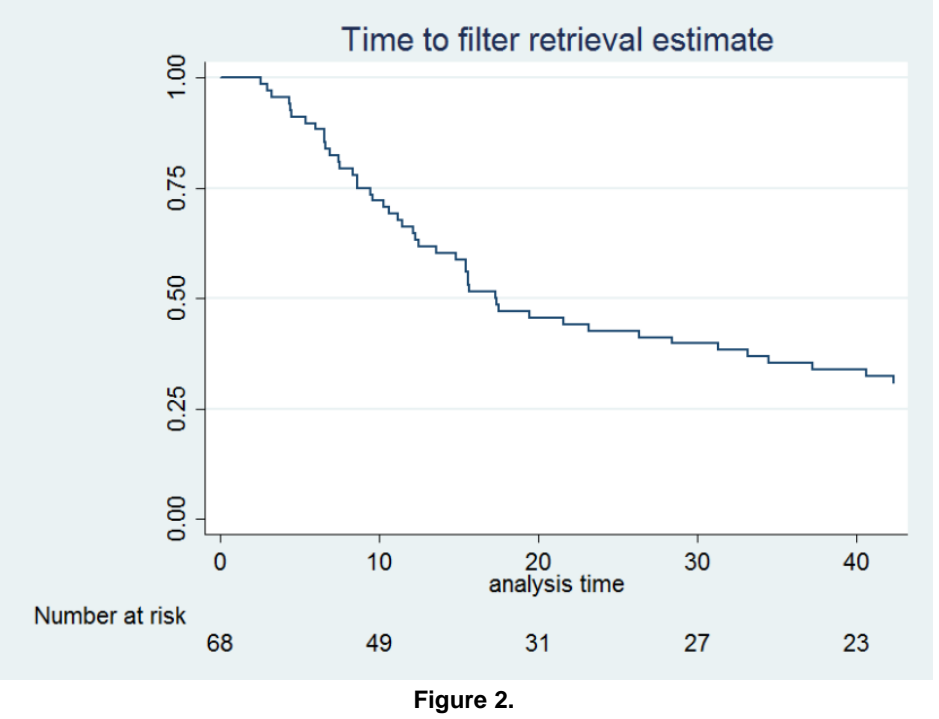

Hematological complications were TED recurrence 30\%, post-thrombotic syndrome $15 \%$ and major bleeding 4.5\%. Twenty-one patients developed a TED recurrence: $22 \%(n=15)$ developed DVT, $8.82 \%(n=6)$ presented PE (Figure 3). Median time to TED recurrence was 40 days (IQR: 9- 173). Hematologic complications had not any statistically significant difference among the subgroups studied (Table 1 . Hematologic complications among different groups and subgroups of patients). When comparing surgeries among different locations, spine fusions were a significant risk factor for developing at least one hematologic complication, especially TED recurrences. When estimating the hazard ratio specifically for TED recurrence adjusted by age and sex, we found no significant association either.

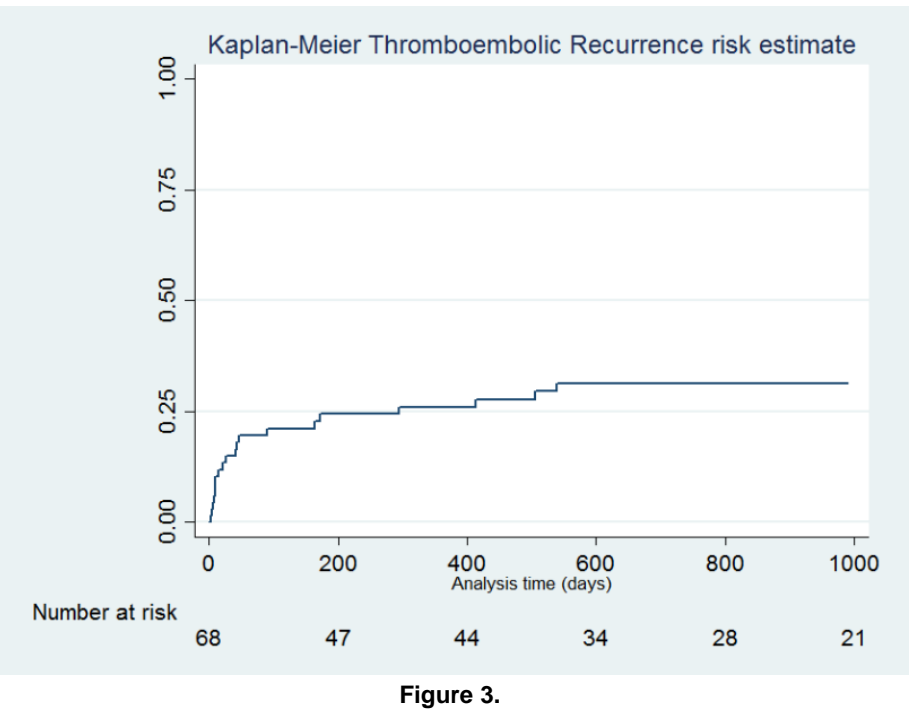

Table 1: Hematologic complications among different groups and subgroups of patients

\begin{tabular}{|lccccc|}
\hline \multicolumn{1}{|c}{ Variable } & Total & $\begin{array}{c}\text { Any } \\
\text { complication }\end{array}$ & Recurrence & syndrome & bleeding \\
\hline Non-arthroplasty & 31 & $51.61(16)$ & $41.94(13)$ & $12.90(4)$ & $6.45(2)$ \\
Arthroplasty & 37 & $37.84(14)$ & $21.62(8)$ & $16.22(6)$ & $2.70(1)$ \\
& & $\mathrm{p}=0.25$ & $\mathrm{p}=0.07$ & $\mathrm{p}=0.70$ & $\mathrm{p}=0.45$ \\
\hline Oncologic & 21 & $57.14(12)$ & $38.10(8)$ & $19.05(4)$ & $14.29(3)$ \\
Non-oncologic & 47 & $38.30(18)$ & $27.66(13)$ & $12.77(6)$ & - \\
& & $\mathrm{p}=0.14$ & $\mathrm{p}=0.38$ & $\mathrm{p}=0.49$ & \\
\hline
\end{tabular}




\begin{tabular}{|c|c|c|c|c|c|}
\hline \multirow{3}{*}{$\begin{array}{l}\text { Primary surgery } \\
\text { Revision surgery }\end{array}$} & & $40.91(18)$ & $34.09(14)$ & $11.36(5)$ & $4.55(2)$ \\
\hline & \multirow{2}{*}{$\begin{array}{l}44 \\
24\end{array}$} & $50 \%(12)$ & $29.17(7)$ & $20.83(5)$ & 4.17 (1) \\
\hline & & $n=047$ & $n=082$ & $n=020$ & \\
\hline \multirow{4}{*}{$\begin{array}{l}\text { Elective surgery } \\
\text { Urgent surgery }\end{array}$} & \multirow{4}{*}{$\begin{array}{l}21 \\
47\end{array}$} & $47.62(10)$ & $33.33(7)$ & $14.29(3)$ & $4.76(1)$ \\
\hline & & & & & \\
\hline & & $42.55(20)$ & $29.79(14)$ & $14.89(7)$ & $4.26(2)$ \\
\hline & & $\mathrm{p}=0.69$ & $\mathrm{p}=0.77$ & $\mathrm{p}=0.94$ & $\mathrm{p}=1$ \\
\hline \multirow{3}{*}{$\begin{array}{l}\text { Preoperative filter } \\
\text { Postoperative filter }\end{array}$} & \multirow{3}{*}{$\begin{array}{l}42 \\
26\end{array}$} & $50(21)$ & $35.71(15)$ & $14.29(6)$ & $7.14(3)$ \\
\hline & & $34.62(9)$ & $23.08(6)$ & $15.38(4)$ & - \\
\hline & & $\mathrm{p}=0.21$ & $p=0.27$ & $\mathrm{p}=0.9$ & - \\
\hline \multirow{6}{*}{$\begin{array}{l}\text { Spinal surgery } \\
\text { Arthroplasty } \\
\text { Rest of the surgeries }\end{array}$} & 9 & $77.78(7)$ & \multirow{3}{*}{$66.67(6)$} & - & $11.11(1)$ \\
\hline & & & & & \\
\hline & 37 & $37.84(14)$ & & $16.22(6)$ & $2.70(1)$ \\
\hline & \multirow[t]{3}{*}{22} & $40.91(9)$ & $21.62(8)$ & $18,18(4)$ & $4.55(1)$ \\
\hline & & & $31.82(7) \mathrm{p}=0.03$ & & \\
\hline & & $\mathrm{p}=0.09$ & & $p=0.40$ & $\mathrm{p}=0.54$ \\
\hline
\end{tabular}

Overall mortality was $28 \%$ ( $n=19)$, involving mostly end-stage oncologic patients ( $68 \%$ of the deceased). The main causes of death were unrelated neither to hematologic complications nor to filter collocation. Seven patients died because of multi-organ failure dysfunction syndrome due to sepsis; 3 due to acute renal failure; 2 because of acute respiratory failure with a negative helical angiotomography for embolism; 2 due to congestive heart failure and 1 patient secondary to a massive abdominal hemorrhage. In 4 cases the cause of death was not reported on the medical charts. Overall mortality due to a certified TED recurrence was $4 \%$. Additionally, $52 \%$ of the deceased (10 out of 19 dead patients) had indication of a VCF because of a history embolic disease whereas on the remaining $48 \%$ it was indicated due to acute or chronic DVT.

\section{Discussion}

This retrospective cohort study provides an in-depth analysis of a group of high-risk patients who had an orthopaedic procedure within the placement of a single-branded retrievable VCF. Overall TED recurrence was $33 \%$, similar to the reported risk for venographic DVT after joint arthroplasty or hip fracture in the absence of anticoagulation, that oscillates between $5 \%$ and $51 \% .{ }^{16-17}$ On the other hand, although overall mortality was $28 \%$, only $4 \%$ of patients died due to a TED recurrence. The reported risk of symptomatic venous thromboembolism (VTE) can be as high as $28 \%$ with up to $10 \%$ of mortality when no thromboprophylaxis is used. ${ }^{18-20}$ Therefore, our findings suggest that with the use of retrievable VCFs, an orthopaedic surgeon may not expect a reduction in the incidence of a new thromboembolic event, but indeed anticipate a decline in the death ratio attributed for TED.

Our study has several limitations. Its retrospective nature correlates with the boundaries of this study design. First, the sample size of the cohort resulted in a small number of patients included in the different groups and subgroups, restraining the production of more accurate statistical analyses such as a multiple regression investigation to categorize independent risk factors. Second, it was an observational with no control group; therefore a prospectively controlled trial remains necessary to corroborate our findings. Third, when evaluating mortality, the cause of death was assessed by the data registered on the digital medical charts and not by autopsy results. Although the circumstances leading to death were reviewed in detail in all cases, a post-mortem examination was not routinely performed due to lack of family consent in many cases, given the inclusion of several end-stage oncologic patients. Therefore, we believe our attributed mortality for TED should be considered of low estimate.

After analyzing 95 joint arthroplasties that received VCFs, Austin et al. ${ }^{21}$ found that filters were effective at preventing fatal PE, although a relatively large number of recurrent DVT occurred, similar to our results. Another study observed an incidence of fatal PE of $4 \%$ with Greenfield permanent filters, recommending its use only in patients who have a thromboembolism with contraindicated anticoagulation, in those with complications secondary to therapeutic anticoagulation and those who are exceptionally at a high-risk for 
thromboembolism. Golueke et al. ${ }^{22}$ reported a similar mortality (4.6\%) in 21 total joint replacements that received a prophylactic preoperative permanent filter.

Thirty percent of our series consisted of oncologic patients. Additionally, of the 19 dead patients, almost $70 \%$ were oncologic. It has been shown that the sole presence of VTE in an otherwise healthy patient should increase surveillance for detecting malignancies. ${ }^{23}$ Oncologic patients definitely have a higher predisposition for thromboembolic events due to multiple causes: extrinsic vascular compression, tumor vascular infiltration, hypercoagulability induced by cancer-related cytokines, and pro-thrombotic chemo and radiotherapies. ${ }^{24-25}$ Mikhail et al. ${ }^{26}$ determined that there was a low overall complication rate after using retrievable VCFs in cancer patients. However, as retrievability was $2 \%$ and the mortality rate was almost $50 \%$ at 90 days, the authors concluded that end-stage patients might not benefit from the use of such filters. Conversely, when examining a group of patients with metastatic pathologic fracture of the lower extremity, a retrospective study evidenced that patients with retrievable VCFs obtained $8 \%$ of DVT and $0 \%$ of PE whereas patients that only received mechanical prophylaxis attained a $4.2 \%$ and $22 \%$ of DVT and PE, respectively. ${ }^{27}$ Given the high mortality rate of the patients that developed PE, the authors strongly recommend the use of VCFs despite the morbidity associated with their insertion.

An inferior VCF's main purpose is to avoid new thromboembolic events in patients in whom anticoagulation has been contraindicated or remains insufficient. Prior studies have reported efficacious results in decreasing the risk of $\mathrm{PE}$ in high-risk trauma patients ${ }^{28}$. Nonetheless, as stated by a large randomized trial of patients with proximal DVT, the initial beneficial effect of VCFs for the prevention of PE seems to be counterbalanced by an excess of recurrent DVT at two years follow-up, without any difference in mortality ${ }^{29}$.

When analyzing permanent filters' long-term effects, the PREPIC randomized controlled trial concluded that at eight years, vena cava filters reduced the risk of PE (hazard ratio 0.37) but increased that of DVT (hazard ratio 1.52) and had no effect on survival. ${ }^{5}$ In order to methodically examine the evidence for the success of VCFs on preventing PE, only 2 studies $^{5,30}$ were able to meet the inclusion criteria in a level I systematic review of the literature carried out by Young et al. ${ }^{31}$ They concluded that both studies lacked statistical power to detect a reduction in PE over shorter and more clinically significant time periods but proved that permanent filters were associated with an increased risk of long-term DVT. Like Young et al., we believe there is very limited evidence concerning VCF outcomes when used within their currently approved indications, especially of retrievable filters.

Several studies have reported on varied percentages of transient filter's mechanical complications and irretrievability when treating orthopaedic patients, especially in trauma cases ${ }^{7,32-38}$. Fullen et al. ${ }^{30}$ reported that technical difficulties, largely mechanical, were more frequent in women and related to the small size of the jugular vein. Nonetheless, they found no filter migration, malposition nor perforation of the surrounding organs. Mechanical hindrances are reported to range from $0 \%$ to $6 \%$ whereas success in retrieving the filter varies from $21 \%$ to $64 \%$. We believe that the true rate of filter retrieval is undervalued, since it can be influenced by the patient's clinical situation, the angiographist's experience and different institutional protocols ${ }^{31}$. We have described one of the highest ratios of mechanical complications. All of them were successfully revised within the short-term. Unfortunately, most of these revised filters (64\%) failed later in obtaining a successful retrieval. Although there in some evidence that the permanent use of a retrievable VCF may be safe in the mid-term,$^{39}$ there is a high likelihood that the long-term settlement of the filter will mechanically stimulate the vessel's endothelium, activating the clotting cascade and enabling its definite incorporation into the wall.

A prospective observational cohort study of symptomatic thromboembolisms in 36388 orthopaedic patients determined an overall low occurrence of VTE (1\%), with the highest incidence observed, in the presence of thromboprophylaxis, after internal fixation of pelvic fractures and total knee replacements; and the highest mortality seen after lower limb amputation and hemiarthroplasties due to hip fractures ${ }^{40}$. Moreover, when comparing 4001 surgical hip procedures, thromboembolic events and mortality were more frequent in THRs and hemiarthroplasties secondary to hip fractures than those performed electively due to degenerative hip disorders or revision surgeries. This concern coincides with our initial hypothesis of expecting more overall complications and mortality rates in patients undergoing arthroplasties, particularly those performed urgently. However, our findings suggest that no differences were found in overall TED recurrence, post-thrombotic syndrome or major bleeding, neither between arthroplasties and non-arthroplasties, nor between urgent and elective procedures. Given that VCFs are usually indicated in extremely high-risk individuals, we consider that our small number of patients is of low estimate to categorize specific risk factors.

We found a trend towards spinal surgeries being associated with TED recurrences. Spinal surgeries proved to have a higher risk of developing thromboembolic events than hip and knee arthroplasties in a previous study, since epidural anesthesia reduced the incidence of VTE when compared to general anesthesia ${ }^{41}$. Major surgical approaches during long general anesthesia periods, extensive spinal decortication and large anatomic dead space created during exposure derives in potential hemorrhagic complications and hematoma formation, which might be counterbalanced by the indication of a VCF. ${ }^{42-43}$ The use of prophylactic VCFs as a standard protocol for high-risk patients undergoing major spinal instrumentations resulted in $18 \%$ 
of patients with DVT and $3.7 \%$ of PE, without any related death in an 8-year period, decreasing the odds of thromboembolic events when compared with population controls ${ }^{44}$. Furthermore, patients that received permanent filters had significantly higher VTE incidence than those receiving retrievable ones. We consider that elderly patients, as the population described within this study, in the setting of long anesthesia-time, plays a major role in the settlement TED-related complications following spinal procedures ${ }^{45-46}$.

In conclusion, orthopaedic procedures had a high risk of mechanical and hematologic complications after using a retrievable VCF in this series of complex patients. However, mortality was low due to these complications.

\section{Bibliography}

1. Colwell CW, Froimson MI, Mont MA, et al. Thrombosis prevention after total hip arthroplasty: a prospective, randomized trial comparing a mobile compression device with low-molecular-weight heparin. $J$ Bone Joint Surg Am 2010; 92: 527-35.

2. Falck-Ytter Y, Francis CW, Johanson NA, et al. Prevention of VTE in Orthopedic Surgery Patients. Chest 2012; 141: e278S-e325S.

3. Dorr LD. Multimodal Thromboprophylaxis for Total Hip and Knee Arthroplasty Based on Risk Assessment. J Bone Jt Surg 2007; 89: 2648.

4. Aziz F, Comerota AJ. Inferior Vena Cava Filters. Ann Vasc Surg 2010; 24: 966-979.

5. Decousus H. Eight-year follow-up of patients with permanent vena cava filters in the prevention of pulmonary embolism: The PREPIC (Prévention du Risque d'Embolie Pulmonaire par Interruption Cave) randomized study. Circulation 2005; 112: 416-422.

6. Bass AR, Mattern CJ, Voos JE, et al. Inferior vena cava filter placement in orthopedic surgery. Am J Orthop (Belle Mead NJ) 2010; 39: 435-9.

7. Strauss EJ, Egol KA, Alaia M, et al. The use of retrievable inferior vena cava filters in orthopaedic patients. J Bone Joint Surg Br 2008; 90: 662-7.

8. Mont $M$ a, Jacobs JJ, Boggio $L N$, et al. Preventing venous thromboembolic disease in patients undergoing elective hip and knee arthroplasty. J Am Acad Orthop Surg 2011; 19: 768-76.

9. Cg L, la I, Sf S. Evaluation of the First-Generation AAOS Clinical Guidelines on the Prophylaxis of Venous Thromboembolic Events in Patients Undergoing Total Joint Arthroplasty. 2014; 1327-1332.

10. Cracowski J, Bosson J, Baloul F, et al. Early development of deep-vein thrombosis following hip fracture surgery : the role of venous wall thickening detected by B-mode ultrasonography. 1998; 269-274.

11. Sharrock NE, Go G, Harpel PC, et al. The John Charnley Award. Thrombogenesis during total hip arthroplasty. Clin Orthop Relat Res 1995; 16-27.

12. Jaff MR, McMurtry MS, Archer SL, et al. Management of massive and submassive pulmonary embolism, iliofemoral deep vein thrombosis, and chronic thromboembolic pulmonary hypertension: a scientific statement from the American Heart Association. Circulation 2011; 123: 1788-830.

13. Kahn SR, Partsch H, Vedantham S, et al. Definition of post-thrombotic syndrome of the leg for use in clinical investigations: A recommendation for standardization. J Thromb Haemost 2009; 7: 879-883.

14. Schulman S, Anger SU, Bergqvist D, et al. Definition of major bleeding in clinical investigations of antihemostatic medicinal products in surgical patients. J Thromb Haemost 2010; 8: 202-204.

15. Liebs TR, Splietker F. Is a Revision a Revision? An Analysis of National Arthroplasty Registries 'Definitions of Revision. Clin Orthop Relat Res 2015; 3421-3430.

16. Geerts WH, Bergqvist D, Pineo GF, et al. Prevention of venous thromboembolism: American College of Chest Physicians evidence-based clinical practice guidelines (8th edition). Chest 2008; 133: 381-453.

17. Lieberman JR, Geerts WH. Prevention of venous thromboembolism after total hip and knee arthroplasty. J Bone Joint Surg Am 1994; 76: 1239-50.

18. Horlander KT, Mannino DM, Leeper K V. Pulmonary embolism mortality in the United States, 1979-1998: an analysis using multiple-cause mortality data. Arch Intern Med 2003; 163: 1711-7.

19. Lapner ST, Kearon C. Diagnosis and management of pulmonary embolism. BMJ 2013; 346: $f 757$.

20. Tagalakis V, Patenaude V, Kahn SR, et al. Incidence of and mortality from venous thromboembolism in a realworld population: the Q-VTE Study Cohort. Am J Med 2013; 126: 832.e13-21.

21. Austin MS, Parvizi J, Grossman S, et al. The inferior vena cava filter is effective in preventing fatal pulmonary embolus after hip and knee arthroplasties. J Arthroplasty 2007; 22: 343-8.

22. Golueke PJ, Garrett W V, Thompson JE, et al. Interruption of the vena cava by means of the Greenfield filter: expanding the indications. Surgery 1988; 103: 111-7.

23. Ferreyro BL, Angriman F, Giunta $D$, et al. Predictive score for estimating cancer after venous thromboembolism: a cohort study. BMC Cancer 2013; 13: 352.

24. Agnelli $G$, Verso $M$, Ageno W, et al. The MASTER registry on venous thromboembolism: Description of the study cohort. Thromb Res 2008; 121: 605-610.

25. Bagot CN, Arya R. Virchow and his triad: A question of attribution. Br J Haematol 2008; 143: 180-190.

26. Mikhail S, Hannan L, Pishvaian MJ, et al. Retrievable inferior vena cava filters in patients with cancer are safe but are they beneficial? Med Oncol 2015; 32: 175.

27. Benevenia J, Bibbo $C$, Patel $D V$, et al. Inferior vena cava filters prevent pulmonary emboli in patients with metastatic pathologic fractures of the lower extremity. Clin Orthop Relat Res 2004; 87-91. 
28. Rogers FB, Strindberg G, Shackford SR, et al. Five-year follow-up of prophylactic vena cava filters in high-risk trauma patients. Arch Surg 1998; 133: 406-11; discussion 412.

29. Decousus H, Leizorovicz A, Parent $F$, et al. A clinical trial of vena caval filters in the prevention of pulmonary embolism in patients with proximal deep-vein thrombosis. Prévention du Risque d'Embolie Pulmonaire par Interruption Cave Study Group. N Engl J Med 1998; 338: 409-15.

30. Fullen WD, Miller EH, Steele WF, et al. Prophylactic vena caval interruption in hip fractures. J Trauma 1973; 13: 403-10.

31. Young T, Tang H, Aukes J, et al. Vena caval filters for the prevention of pulmonary embolism. Cochrane Database Syst Rev. Epub ahead of print 2007. DOI: 10.1002/14651858.CD006212.pub3.

32. Albrecht RM, Garwe T, Carter SM, et al. Retrievable inferior vena cava filters in trauma patients: Factors that influence removal rate and an argument for institutional protocols. Am J Surg 2012; 203: 297-302.

33. Allen TL, Carter JL, Morris BJ, et al. Retrievable vena cava filters in trauma patients for high-risk prophylaxis and prevention of pulmonary embolism. Am J Surg 2005; 189: 656-61.

34. Antevil JL, Sise MJ, Sack DI, et al. Retrievable Vena Cava Filters for Preventing Pulmonary Embolism in Trauma Patients: A Cautionary Tale. J Trauma Inj Infect Crit Care 2006; 60: 35-40.

35. Cherry $R$ a, Nichols $P$ a, Snavely TM, et al. Prophylactic inferior vena cava filters: do they make a difference in trauma patients? J Trauma 2008; 65: 544-548.

36. Johnson ON, Gillespie DL, Aidinian G, et al. The use of retrievable inferior vena cava filters in severely injured military trauma patients. J Vasc Surg 2009; 49: 410-416.

37. Karmy-Jones R, Jurkovich GJ, Velmahos GC, et al. Practice patterns and outcomes of retrievable vena cava filters in trauma patients: an AAST multicenter study. J Trauma 2007; 62: 17-24-5.

38. Rosenthal $D$, Wellons ED, Lai KM, et al. Retrievable inferior vena cava filters: initial clinical results. Ann Vasc Surg 2006; 20: 157-65.

39. Myojo M, Takahashi M, Tanaka T, et al. Midterm Follow-up After Retrievable Inferior Vena Cava Filter Placement in Venous Thromboembolism Patients With or Without Malignancy. Clin Cardiol 2015; 38: 216-221.

40. Lapidus LJ, Ponzer S, Pettersson H, et al. Symptomatic venous thromboembolism and mortality in orthopaedic surgery - an observational study of 45968 consecutive procedures. BMC Musculoskelet Disord 2013; 14: 1.

41. Charen DA, Qian ET, Hutzler LH, et al. Risk Factors for Postoperative Venous Thromboembolism in Orthopaedic Spine Surgery, Hip Arthroplasty, and Knee Arthroplasty Patients. 2015; 73: 198-203.

42. Ozturk C, Ganiyusufoglu K, Alanay A, et al. Efficacy of prophylactic placement of inferior vena cava filter in patients undergoing spinal surgery. Spine (Phila Pa 1976) 2010; 35: 1893-6.

43. Rosner MK, Kuklo TR, Tawk R, et al. Prophylactic placement of an inferior vena cava filter in high-risk patients undergoing spinal reconstruction. Neurosurg Focus 2004; 17: E6.

44. McClendon J, O'shaughnessy B a, Smith TR, et al. Comprehensive assessment of prophylactic preoperative inferior vena cava filters for major spinal reconstruction in adults. Spine (Phila Pa 1976) 2012; 37: 1122-9.

45. Masuda K, Chikuda H, Yasunaga H, et al. Factors affecting the occurrence of pulmonary embolism after spinal surgery: data from the national administrative database in Japan. Spine J 2012; 12: 1029-34.

46. Takai $N$, Takasugi $Y$, Kajikawa $R$, et al. Fatal pulmonary embolism following spinal surgery in a patient with permanent inferior vena cava filter placement. J Anesth 2014; 28: 606-609. 